\title{
Actitudes ambientales en jóvenes del nivel medio superior
}

\section{Environmental attitudes in young people of the upper middle level}

\author{
DOI: $10.46932 / s f j d v 3 n 1-124$
}

Received in: January $20^{\text {th }}, 2022$

Accepted in: February $1^{\text {st }}, 2022$

\section{Catalina Vargas Ramos}

Doctora

Institución: Universidad Autónoma de Tamaulipas

Dirección: Ariano Matamoros s/n, Zona Centro, 87000 Cd Victoria, Tamps., México

Correio electrónico: cvargasr@docentes.uat.edu.mx

\section{María Guadalupe Martínez Treviño}

Institución: Universidad Autónoma de Tamaulipas

Dirección: Ariano Matamoros s/n, Zona Centro, 87000 Cd Victoria, Tamps., México

Correio electrónico: mgmtrevino@docentes.uat.edu.mx

\section{RESUMEN}

A medida que avanzamos la población se encuentra bajo el llamado "dilema ambiental (A la Torres, Zavala \& Alvarado, 2014) que ocasiona la pérdida de suelos, de recursos hídricos, contaminación de residuos sólidos peligrosos y no peligrosos, contaminación visual, cambio climático, deforestación y pérdida de la biodiversidad, afectando todos los ecosistemas (Espejel \& Flores, 2012). Por lo que una de las estrategias para dar solución a este dilema es la educación ambiental (EA), ya que es una herramienta que ayuda a adquirir conciencia, valores, conducta (actitudes) y estilos de vida, así como ampliar conocimientos para impulsarlos a la acción mediante la prevención y mitigación de los problemas existentes y futuros. Por ello el objetivo de esta investigación es conocer el nivel de las actitudes que poseen los jóvenes del nivel medio superior, esto por medio de la aplicación del instrumento, "Un pequeño examen de conciencia ambiental" a estudiantes del nivel medio superior.

Palabras clave: actitudes pro ambientales, educación ambiental, dilema ambiental, cambio climático.

\begin{abstract}
As we move forward, the population is under the so-called "environmental dilemma (A la Torres, Zavala $\&$ Alvarado, 2014) that causes the loss of soil, water resources, contamination of hazardous and nonhazardous solid waste, visual pollution, climate change, deforestation and loss of biodiversity, affecting all ecosystems (Espejel \& Flores, 2012). Therefore, one of the strategies to solve this dilemma is environmental education (EA), since it is a tool that helps to acquire awareness, values, behavior (attitudes) and lifestyles, as well as expand knowledge to encourage them to action by preventing and mitigating existing and future problems. For this reason, the objective of this research is to know the level of attitudes that young people of the upper secondary level possess, this through the application of the instrument, "A small environmental conscience examination" to students of the upper secondary level.
\end{abstract}

Keywords: pro-environmental attitudes, environmental education, environmental dilemma, climate change. 


\section{INTRODUCCIÓN}

A medida que avanzamos la población se encuentra bajo el llamado "dilema ambiental", siendo el resultado de cuatro factores: el incremento de la población, contaminación, exceso en el consumo de los recursos y el deterioro de la ética de la Tierra. (A la Torres, Zavala \& Alvarado, 2014)

Por lo que la problemática ambiental actual va desde la pérdida de suelos, de recursos hídricos, contaminación de residuos sólidos peligrosos y no peligrosos, contaminación visual, cambio climático, deforestación y pérdida de la biodiversidad, afectando todos los ecosistemas (Espejel \& Flores, 2012). El margen de acción, no es muy amplio, por lo que en cuestiones de décadas (Isaac-Márquez, Salavarría, Eastmond, Ayala, Arteaga, Isaac- Márquez, A., Sandoval \& Manzanero, 2011) la perdida de los recursos naturales renovables y no renovables, será irreversible. (Espejel \& Flores, 2012)

Por ello una de las primeras soluciones es la participación de la población, por lo que una de las estrategias es la educación ambiental (EA), siendo la herramienta elemental para que todas las personas adquieran conciencia de la importancia de preservar su entorno y sean capaces de realizar cambios en sus valores, conducta (actitudes) y estilos de vida, así como ampliar sus conocimientos para impulsarlos a la acción mediante la prevención y mitigación de los problemas existentes y futuros (Espejel \& Flores, 2012)

Así mismo la educación ambiental tuvo su origen entre los años 70`s, ya que se tenía la preocupación por el deterioro ambiental que iniciaba, por el desarrollo tecnológico y la severa desestabilización de los recursos naturales que se estaba presentando (Alea, 2005) debido a las conductas humanas mal adaptadas al abuso de los mismos. (Martínez, 2004)

Por lo que a finales de los setentas la Organización de las Naciones Unidas para la Educación, la Ciencia y la Cultura realizaron un gran esfuerzo para lograr que la educación ambiental se llevara a cabo en las escuelas como estrategia pedagógica, para hacer una conciencia y formar una responsabilidad humana en la cual se mantuvieran en mente los diferentes tipos de vida en el planeta y la problemática ambiental. (Calixto, 2012).

Sin embargo la falta de educación ambiental en las personas en general, ha provocado grandes problemas ambientales generado un severo impacto dentro de nuestro planeta, principalmente por la falta de conocimiento, educación, practica, actitudes y compromisos ambientales por parte de la sociedad teniendo como resultado de todos estos actos de irresponsabilidad humana; el cambio climático, el efecto invernadero, la pérdida de biodiversidad y la erosión entre otros (Vargas, Vázquez, Gutiérrez, Medellín \& Velázquez, 2012). Siendo la falta de educación el primer problema que causa la problemática medioambiental la cual se puede dividir en dos grandes grupos, la depredación de los recursos naturales y la contaminación que estos mismos generan (Vargas, Rivera, Gutiérrez, Vázquez, Fernández \& Zamorano, 2013). 
Por eso la importancia de la educación ambiental al incorporarla al currículo en las instituciones, para poder formar ciudadanos interesados en el impacto que generan sus acciones. (A la Torres, Zavala \& Alvarado, 2014).

Y es desde 1977, en la conferencia intergubernamental de Tbilisi, que se acordó agregar la educación ambiental al sistema educativo, para tratar de modificar las actitudes, dar conocimiento y motivar la participación de la sociedad, para dar búsqueda a una solución a los problemas ambientales. (Maldonado, 2005)

Sin embargo, es debido a la falta de conocimiento, educación, práctica, actitudes y compromisos ambientales que los seres humanos podríamos perder muchos de nuestros recursos naturales, ya que todos los elementos del medio ambiente están relacionados y si alguno de ellos se viera afectado por consecuencia se alteran los siguientes, debido a la cadena alimenticia. (Vargas, Rivera, Gutiérrez, Vázquez, Fernández \& Zamorano, 2013).

Por esta necesidad y a partir del proyecto de los objetivos de la Educación ambiental, se han realizado una serie de seminarios y congresos en diferentes partes del mundo para promover la divulgación de la educación ambiental como una herramienta fundamental para la sustentabilidad del ambiente. (Espejel \& Flores, 2012)

Por lo tanto, como citan Vargas, Medellín, Vázquez \& Gutiérrez (2011) en una de sus investigaciones, es de vital importancia el fomento de la conciencia y conocimiento ambiental en las generaciones futuras produciendo así un equilibrio entre el hombre y la naturaleza.

Como consecuencia Villegas \& López (2006) hacen una observación para la formación a nivel superior para que los estudiantes tengan un compromiso verdadero y una responsabilidad ante la sociedad.

Sin embargo, las actitudes pro ambientales y las conductas responsables ecológicamente hablando, están muy bajas, ya que el incremento de la sensibilidad en las personas de los países desarrollados, parece no haberse encontrado aún. (Álvarez \& Vega, 2009).

De acuerdo con algunas teorías y especificando sobre la Teoría de Acción Ambiental Positiva de Emmons la cual fue mostrada en el año 1997, nos plasma que el comportamiento humano está basado en el conocimiento ambiental, los valores ambientales, la sensibilidad y las actitudes positivas que se tienen a nuestro medio ambiente, y se llevan a cabo mediante las habilidades y los procedimientos personales que cada una de las personas realizan frente a situaciones que necesiten mostrar esta sensibilidad hacia nuestro medio ambiente para que no se pierda esta relación que existe entre hombre-ambiente. Y para calificar una actitud se deben atender las siguientes cuestiones: los conocimientos y creencias que el individuo tiene sobre el objeto de la actitud, la disposición que muestra el individuo tras realizar una dirección determinada por los aspectos afectivos que lo llevan a tener una actitud ya sea positiva o negativa 
y por último la conducta que el individuo muestra ante alguna situación ya determinada y que conlleva a tener un resultado de actitud. (Hernández \& Jiménez2010).

Y existen estrategias de educación ambiental, en las cuales se proponen cinco pasos para llevarlas a cabo. El primero se dirige realizar un diagnóstico en el que la comunidad determine sus conocimientos, intereses y necesidades ambientales, después proseguir con la elaboración de un proyecto de acuerdo a las necesidades que se mencionaron anteriormente e invitar a exponentes especializados en el tema, tener calendarizado el evento y por último tener una Evaluación. Y por eso la educación ambiental se toma como una herramienta importante para lograr un México con un desarrollo sustentable bien definido y así poder llegar a tener una bien y buena conciencia ambiental. (Vargas, Vázquez, Gutiérrez, Vargas \& Fernández, 2010)

\section{METODOLOGÍA}

Este tipo de estudio según Vargas, Vázquez, Gutiérrez, Medellín \& Velázquez (2012), es cuantitativo descriptivo ya que para obtener los resultados se mide solamente una variable.

Esta investigación fue realizada con una muestra de 120 alumnos del Centro de Bachillerato Tecnológico e Industrial No.135 del turno vespertino, en el primer semestre, tercer semestre y quinto semestre de las carreras: Logística, Recursos Humanos y Electrónica, en un horario que abarco entre las 15:00 - 16:00 hrs., un rango de edad entre los 15 años y 18 años y de ambos sexos.

La información recopilada en esta investigación fue por medio del instrumento llamado "Un pequeño examen de conciencia ambiental", el cual fue aplicado con anterioridad en una investigación científica la cual tiene por nombre "Cambio en actitudes ambientales en estudiantes del nivel superior ante el impacto de la asignatura de desarrollo sustentable" (Vargas, Vázquez, Gutiérrez, Medellín \& Velázquez, 2012).

Dicho instrumento cuenta con 16 items de respuesta tipo liker, el cual mide cuatro dimensiones ambientales: a) Consumista derrochador, siendo la acumulación de bienes y servicios no esenciales, b) Consumista consciente, es la acción de compra bienes no tan esenciales y se pueden suprimir sin ninguna consecuencia, c) Ecologista bien encaminado, que es el que procura el cuidado del medio ambiente, pero no reflexiona ante el impacto generado, d) Ecologista cuidado con la madre Tierra, reflexiona ante el impacto que genera en sus acciones y hace uso de sus valores, actitudes ambientales.

El instrumento tiene respuestas que van desde Nunca, Casi siempre y Siempre con un valor de $1-$ 3 respectivamente.

Además, se valoraron variables socio-demográficas, las cuales fueron: sexo, edad, especialidad y semestre. 
La escala que se utilizó en esta investigación para medir las actitudes ambientales está dividida en cuatro categorías: consumista derrochador, consumista consciente, ecologista bien encaminado y ecologista cuidadoso con la madre siendo esta la mayor, como también se presentan los rangos que se les asignaron a cada una de las categorías y esto se presenta a continuación (Ver tabla 1)

Tabla 1 Rango y categorías de actitudes ambientales

\begin{tabular}{ll}
\hline \multicolumn{1}{c}{ NIVEL DE ACTITUD AMBIENTAL } & Rango \\
\hline Consumista Derrochador & $1-11$ \\
Consumista Consciente & $12-22$ \\
Ecologista Bien encaminado & $23-30$ \\
Ecologista Cuidadoso con la madre Tierra & $31-33$ \\
\hline
\end{tabular}

\section{PROCEDIMIENTO}

La encueta se aplicó a tres diferentes grupos de diferente especialidad: logística, recursos humanos y electrónica, de 1er semestre, 3er semestre y 5to semestre respectivamente, para lo cual se solicitó la aprobación para acudir a las aulas para la recolección de los datos, se les leyeron las instrucciones y se procedió al llenado. El tiempo de aplicación en cada grupo fue de 10 - 15 minutos aproximadamente, el llenado de las encuestas fue anónimo participando todos los alumnos de cada grupo teniendo un rango de edades de entre los 15 - 18 años. El instrumento contiene 16 items de tipo Likert siendo: Nunca, Casi siempre y Siempre con un valor de $1-3$ respectivamente.

\section{RESULTADOS}

De acuerdo a la información recopilada por medio de las encuestas se obtuvieron los siguientes resultados:

En cuanto a los resultados por genero se encontró que solo el $3 \%$ del sexo femenino tiene una actitud ambiental alta, debido que están en la categoría de Ecologista cuidadoso con la madre tierra, y solo el $2 \%$ del sexo masculino en la misma categoría, pero se observa que el mayor porcentaje está en la categoría de Consumista Consciente con un 63\% del sexo femenino. (Ver tabla 2)

Tabla 2. Nivel de actitud ambiental según el sexo.

\begin{tabular}{llcc}
\hline NIVEL DE ACTITUD AMBIENTAL & Femenino (67) & Masculino (53) \\
\hline $\mathbf{1 - 1 1}$ & CONSUMISTA DERROCHADOR & $1 \%$ & $2 \%$ \\
$\mathbf{1 2 - 2 2}$ & CONSUMISTA CONSCIENTE & $63 \%$ & $58 \%$ \\
$\mathbf{2 3 - 3 0}$ & ECOLOGISTA BIEN ENCAMINADO & $33 \%$ & $38 \%$ \\
$\mathbf{3 1 - 3 3}$ & ECOLOGISTA CUIDADOSO CON LA MADRE TIERRA & $3 \%$ & $2 \%$ \\
\hline
\end{tabular}

También se puedo observar que de acuerdo en la especialidad se obtuvo un alto porcentaje en la categoría de Ecologista cuidadoso con la madre tierra, siendo esta la categoría mayor fue la carrera de 
Recursos Humanos con un 6\%, pero la carrera de Electrónica que obtuvo el mismo 6\% pero en la categoría de Consumista Derrochador (Ver tabla 3)

Tabla 3 Nivel de actitud ambiental en especialidades.

\begin{tabular}{lcccc}
\hline ESPECIALIDAD & $\begin{array}{l}\text { CONSUMISTA } \\
\text { DERROCHADOR }\end{array}$ & $\begin{array}{l}\text { CONSUMISTA } \\
\text { CONSCIENTE }\end{array}$ & $\begin{array}{l}\text { ECOLOGISTA } \\
\text { BIEN } \\
\text { ENCAMINADO }\end{array}$ & $\begin{array}{l}\text { ECOLOGISTA } \\
\text { CUIDADOSO } \\
\text { CON LA MADRE } \\
\text { TIERRA }\end{array}$ \\
\hline ELECTRONICA & $6 \%$ & $57 \%$ & $37 \%$ & $0 \%$ \\
RECURSOS & $0 \%$ & $62 \%$ & $32 \%$ & $6 \%$ \\
HUMANOS & & & & $0 \%$ \\
LOGISTICA & $0 \%$ & $62.5 \%$ & $37.5 \%$ & 0 \\
\hline
\end{tabular}

Y de acuerdo con el semestre, se tiene que en la categoría de Ecologista cuidadoso con la madre tierra, el primer semestre cuenta con el 6\%, siendo este el único con porcentaje, pero en cambio el quinto semestre el $6 \%$ se encuentra en la categoría de consumista derrochador lo cual es preocupante. (Ver tabla 4)

Tabla 4 Nivel de actitud ambiental por semestres.

\begin{tabular}{ccccc}
\hline SEMESTRE & $\begin{array}{c}\text { CONSUMISTA } \\
\text { DERROCHADOR }\end{array}$ & $\begin{array}{c}\text { CONSUMISTA } \\
\text { CONSCIENTE }\end{array}$ & $\begin{array}{c}\text { ECOLOGISTA } \\
\text { BIEN } \\
\text { ENCAMINADO }\end{array}$ & $\begin{array}{c}\text { ECOLOGISTA } \\
\text { CUIDADOSO } \\
\text { CON LA MADRE } \\
\text { TIERRA }\end{array}$ \\
\hline to & $6 \%$ & $57 \%$ & $37 \%$ & $0 \%$ \\
$3 \mathrm{er}$ & $0 \%$ & $62.5 \%$ & $37.5 \%$ & $0 \%$ \\
$1 \mathrm{er}$ & $0 \%$ & $62 \%$ & $32 \%$ & $6 \%$ \\
\hline
\end{tabular}

Y por último la edad promedio de los alumnos que tuvieron mayor actitud ambiental de acuerdo a la categoría Ecologista cuidadoso con la madre Tierra corresponde a la población que tiene 16 años con un porcentaje de $12.5 \%$, por el contrario, con un $73 \%$ los que tuvieron menor actitud ambiental fue en la categoría de consumista consciente oscila entre los 17 años. (Ver tabla 5)

Tabla 5 Nivel de actitud ambiental de acuerdo a la edad.

\begin{tabular}{ccccc}
\hline EDAD & $\begin{array}{c}\text { CONSUMISTA } \\
\text { DERROCHADOR }\end{array}$ & $\begin{array}{c}\text { CONSUMISTA } \\
\text { CONSCIENTE }\end{array}$ & $\begin{array}{c}\text { ECOLOGISTA BIEN } \\
\text { ENCAMINADO }\end{array}$ & $\begin{array}{c}\text { ECOLOGISTA } \\
\text { CUIDADOSO CON LA } \\
\text { MADRE TIERRA }\end{array}$ \\
15 & $0 \%$ & $68 \%$ & $30 \%$ & $2 \%$ \\
16 & $0 \%$ & $25 \%$ & $62.5 \%$ & $12.5 \%$ \\
17 & $0 \%$ & $73 \%$ & $27 \%$ & $0 \%$ \\
18 & $0 \%$ & $33 \%$ & $67 \%$ & $0 \%$ \\
\hline
\end{tabular}




\section{CONCLUSIONES}

Con respecto a los resultados obtenidos en esta investigación que fue realizada a jóvenes del nivel medio superior del Centro de Bachillerato Tecnológico Industrial y de Servicios \# 135, se puede observar que existe muy poca población estudiantil que cumple con la categoría de Ecologista Comprometido con la Madre Tierra, siendo esta la máxima categoría ambiental, observándose un mayor porcentaje en la categoría de Consumista Consciente, esto coincide con los resultados que obtuvieron Vargas, Rivera, Gutiérrez, Vázquez, Fernández \& Zamorano, en el 2013, donde nos menciona que los estudiantes a nivel licenciatura tienen un nivel de educación Inaceptable.

Además, Sosa, en el 2010, también realizo una investigación en la Universidad Autónoma de Campeche, donde al igual que esta investigación la población estudiantil obtuvo un bajo nivel de cultura - ambiental, por lo tanto, carecían de conocimientos y de habilidades que son necesarias para llevar una actitud ambiental favorable para el medio ambiente.

También Montaño, Cervantes, Gutiérrez \& Zarate (2012), tomando el tema sobre el Nivel de alfabetización ambiental y utilizando un instrumento diferente obtuvo mejores resultados ya que con esa investigación el demostró la población estudiantil tiene actitudes ambientales aceptable, pero por el contrario en conocimientos y comportamientos indico que el resultado fue inaceptable.

Evaluando los diferentes resultados se observa y se puede referir que existen actitudes desfavorables con respecto al medio ambiente en la población estudiantil en el nivel medio superior y en el nivel superior, cabe mencionar que esta investigación fue realizada a jóvenes del nivel medio superior lo cual nos da un margen de formación buena para corregir estas actitudes que desfavorecen al medio ambiente, se recomienda en exista un mayor compromiso tanto de los docentes como de los alumnos en cuestión de la realización de talleres, prácticas ambientales, platicas en las cuales se les brinde información que les sirva para darse cuenta de las desventajas de tener una actitud desfavorable con el ambiente, y así poder abrir su mente ante las consecuencias que pudieran surgir y cambiar su forma de pensar, sus actitudes y su comportamiento ante el medio ambiente y que tomen conciencia ante todas las circunstancias que pudieran ocurrir, como lo recomienda Vargas, Rivera, Gutiérrez, Vázquez, Fernández \& Zamorano (2013). 


\section{REFERENCIAS}

A la Torre, M., Zavala, A. \& Alvarado, J. (2014). Alfabetización ambiental en estudiantes de ingeniería de la Universidad de Sonora. Revista Epistemus. Vol. 16(8). pp.79-87. Disponible en: http://www.epistemus.uson.mx/revistas/articulos/16-art11_ALFABETIZACIONAMBIENTAL.pdf

Álvarez, P. \& Vega, P. (2009). Actitudes ambientales y conductas sostenibles. Implicaciones para la educación ambiental. Revista de Psicodidáctica. Vol. 14(2). pp. 24-260. Disponible en: http://www.redalyc.org/pdf/175/17512724006.pdf

Andrade, M. (2011). Estado del conocimiento de la biodiversidad en Colombia y sus amenazas. Consideraciones para fortalecer la interacción Ciencia-Política. Rev. Acad. Colomb. Cienc. Vol. Xv (137). pp. 492-507. Disponible en:

\section{http://accefyn.org.co/revista/Vol_35/137/492-508.pdf}

Baños, M., González, N. \& Álvarez, J. (2013). Cambio de actitud proambiental en estudiantes de bachillerato, en México. Revista de Didáctica Ambiental. Vol. 9(12). pp. 1-12. Disponible en línea: http://www.didacticaambiental.com/revista/revista12/articulos\%20PDF/1.actitud\%20proambiental\%20c opia.pdf

Calixto, R. \& Herrera, L. (2010). Estudio sobre las percepciones y la educación ambiental. Tiempo de Educar. Vol. 11(22), pp. 227-249. Disponible en:

\section{http://www.redalyc.org/pdf/311/31121072004.pdf}

Elvira, J (2009). SEMARNAT. Cambio climático. Ciencia, evidencia y acciones. Disponible en:http://www.semarnat.gob.mx/archivosanteriores/informacionambiental/Documents/05_serie/cambio_ climatico.pdf

Espejel, A., Castillo, I. \& Martínez, H. (2011). Modelo de educación ambiental para el nivel medio superior, en la región Puebla-Tlaxcala, México: un enfoque por competencias. Revista Iberoamericana de Educación. n 55/4. pp 2-13. Disponible en:

\section{http://www.rieoei.org/expe/3705Espejel.pdf}

Espejel, A. \& Flores, A. (2012). Educación ambiental escolar y comunitaria en el nivel medio superior, Puebla - Tlaxcala, México. Revista mexicana de investigación educativa. Vol. 17. (55). Disponible en: http://www.scielo.org.mx/scielo.php?pid=S1405-66662012000400008\&script=sci_arttext

Gomera, A., Villamandos, F. \& Vaquero, M. (2012). Medición y categorización de la conciencia ambiental del alumnado universitario: contribución de la Universidad a su fortalecimiento. Revista de Currículum y formación del profesorado. Vol. 16 (2). pp. 214-228. Disponible en: http://www.ugr.es/ recfpro/rev162ART11.pdf

Isaac- Márquez, R., Salavarría, O., Eastmond, A., Ayala, M. Arteaga, M., Isaac-Márquez, A., Sandoval, J. \& Manzanero, L. (2011). Cultura ambiental en estudiantes de bachillerato. Estudio de caso de la educación ambiental en el nivel medio superior de Campeche. Revista Electrónica de Investigación Educativa. Vol. 13(2). pp. 84- $98 . \quad$ Disponible en línea: http://scielo.unam.mx/pdf/redie/v13n2/v13n2a6.pdf

Maldonado, H. (2005). La educación ambiental como herramienta social. Revista Geoenseñanza. Vol. 10(1). pp.61-67. Disponible en: http://www.saber.ula.ve/bitstream/123456789/21003/2/articulo4.pdf 
Martínez, J. (2004). La estructura de la responsabilidad proambiental hacia la conservación de agua potable en jóvenes estudiantes. Revista Medio Ambiente y Comportamiento Humano. Vol. 5. (1y2). Pp. 115-132. Disponible en línea: http://mach.webs.ull.es/PDFS/Vol5_1y2/VOL_5_1y2_f.pdf

Montaño, F., Cervantes, A., Gutiérrez, M. \& Zarate, M. (2012). Nivel de alfabetización ambiental en estudiantes de Ingeniería en ciencias ambientales del Instituto Tecnológico de Sonora. DELOS: Desarrollo Local Sostenible. Vol. 5 (14). Disponible en:

http://www.eumed.net/rev/delos/14/sbco.html

Sánchez, F. \&Yera, D. (2012). El fortalecimiento de la educación ambiental mediante talleres de apreciación-creación en el 4to grado de la escuela Conrado Benítez. DELOS: Desarrollo Local Sostenible. Vol. 5 (15). Disponible en:

http://www.eumed.net/rev/delos/15/sgyj.html

Santiago, J. (2008). La problemática del ambiente, la educación ambiental y el uso didáctico de los medios de comunicación social. Investigación y Postgrado. Vol.23 (2). Disponible en: http://www.scielo.org.ve/scielo.php?pid=S1316-00872008000200009\&script=sci_arttext

Sosa, S., Márquez, R., Eastmond, A., Ayala, M. \& Arteaga M. (2010). Educación superior y cultura ambiental en el sureste de México. Revista: Universidad y Ciencia. Vol. 26 (1). pp. 33-49. Disponible en: http://www.redalyc.org/articulo.oa?id=15416251003

Tovar, J. (2012). Hacia una educación ambiental ciudadana contextualizada: consideraciones teóricas y metodológicas. Desde el trabajo por proyectos. Revista Iberoamericana de Educación. n 58/2. pp 2-11. Disponible en: http://www.rieoei.org/expe/4322Tovar.pdf

Vargas, C., Briones, C., Mancha, M., Múzquiz, P. \& Vargas, A. (2013). Conciencia ambiental de los habitantes de la colonia Emilio Portes Gil en la H. Matamoros, Tamaulipas. Luna Azul. Vol (37). Disponible en:

\section{http://www.scielo.org.co/scielo.php?pid=S1909-24742013000200011\&script=sci_arttext}

Vargas, C., Medellín, J., Vázquez, L. \& Gutiérrez, G. (2011) Actitudes ambientales en los estudiantes de nivel superior en México. Luna Azul. Vol. (33). Disponible en:

http://www.scielo.org.co/scielo.php?pid=S1909-24742011000200004\&script=sci_arttext

Vargas, C., Rivera, F., Gutiérrez, G., Vázquez, L., Fernández, M. \& Zamorano, B. (2013). Nivel de educación ambiental a través de las actitudes, comportamientos y conocimientos ambientales en el nivel de Licenciatura., Revista Universitaria de Investigación y Diálogo Académico. Vol. 9 (1). pp 63-72. Disponible en:

http://conhisremi.iuttol.edu.ve/pdf/ARTI000174.pdf

Vargas, C., Vázquez, L., Gutiérrez, G., Medellín, J. \& Velázquez, Y. (2012). Cambio en actitudes ambientales en estudiantes del nivel superior ante el impacto de la asignatura de Desarrollo Sustentable. Revista Universitaria de Investigación y Dialogo Académico. Vol. 8 (2). 47-59. Disponible en:

http://conhisremi.iuttol.edu.ve/pdf/ARTI000153.pdf 
Vargas, C., Vázquez, L., Gutiérrez, G., Vargas, A. \& Fernández, C. (2010). Conciencia ambiental en estudiantes de Enfermería Básica. Revista Universitaria de Investigación y Diálogo Académico. Vol. 6(3). Pp. 18-28. Disponible en: http://conhisremi.iuttol.edu.ve/pdf/ARTI000103.pdf

Vega, P. \& Álvarez, P. (2005). Planteamiento de un marco teórico de la Educación Ambiental para un desarrollo sostenible. Revista Electrónica de Enseñanza de las Ciencias. Vol. 4 (1). 94 pp. Disponible en:

http://reec.uvigo.es/volumenes/volumen4/ART4_Vol4_N1.pdf

Vega, P., Freitas, M., Álvarez, P. \&Fleuri, R. (2007). Marco teórico y metodológico de educación ambiental e intercultural para un desarrollo sostenible. Revista Eureka sobre Enseñanza y Divulgación de las Ciencias. Vol. 4(3). pp. 539-554. Disponible en:

http://www.ced.ufsc.br/ mover/memorialfleuri/ap2007_EUREKA_VEGA_FREITAS_ALVAREZ_FLE URI_Desarollo_sostenible.pdf 